\title{
Buenos Aires dans les Années 90: Gouvernance Urbaine dans une Métropole Divisée
}

\author{
M arie-France Prévôt Schapira*
}

Resumo: 0 que significa falar de governança urbana, em uma metrópole de 12 milhões de habitantes na qual se acentuou, durante os anos 90, a divisão entre 0 centro da cidade dopado pela nova economia e as imensas periferias devastadas pela pobreza epelo desemprego? Essa análise sobre a governança na aglomeração de Buenos Aires mostra que a idéia metropolitana é confrontada às fronteiras políticas e sociais que dividem este espaço fragmentado. Aqui os diferentes estilos de governança são analisados por um lado, a partir da estrutura espacial e da arquitetura política da metrópole que sobre- determinam as gestões locais e por outro lado, a partir da capacidade dos territórios de se transformarem em atores coletivos da governança e influnciarem sua evolução.

Palavras-Chave: Governança Urbana, Cidades, Gestão local

Résume: Que signifie parler de gouvernance urbaine, dans une grande métropole de 12 millions d'habitants qui a vu, dans les années 90 , se creuser la coupure entre la ville-centre dopée par la nouvelle économie et les immenses périphéries dévastées par la pauvreté et le chômage? Cette analyse sur la gouvernance dans l'agglomération de Buenos Aires montre que l'idée métropolitaine vient buter sur les frontières politiques et sociales qui divisent cet espace fragmenté. Les différents styles de gouvernance sont ici analysés à partir d'une part, de la structure spatiale et l'architecture politique de la métropole qui surdéterminent les gestions locales et d'autre part, de la capacité des territoires à devenir des acteurs collectifs de la gouvernance et à peser sur leur devenir.

Mots-Clés: Gouvernance Urbaine, Villes, Gestions Locales.

*Professeur de Géografie à L'Université Paris 8. E-mail: schapira@ ivry.cnrs.fr 
La mondialisation économique signifiel'accroissement de la mobilité du capital et la possibilité pour les capitaux de s'affranchir des contraintes spatiales. Paradoxalement, cet affranchissement va de pair avec une sensibilité accrue au territoire, notamment aux villes, en tant que lieu d'investissement et de vie dominants (Schapira 2001). Il existe désormais une convergence nouvelle entre chercheurs, opérateurs et décideurs, au N ord comme au Sud, pour considérer quela ville est un moteur essentiel du développement et qu'en conséquence, I'urbanisation constitue un défi majeur pour le développement durable $e^{1}$.

Pour l'Amérique latine, ce prédicat a une importance majeure car la population urbaine est largement majoritaire. Les villes regroupent désormais les trois quarts dela population. D urant la période substitutive se sont les grandes villes qui ont connu des rythmes de croissance sans précédent dans l'histoire. Ce rythmes'infléchit à partir des années 80, notamment dans les grandes villes. Toutefois, partout l'accent est mis sur le rôle majeur du processus de métropolisation induit par la mondialisation croissante de l'économie et sur la restructuration profonde de la hiérarchie urbaine mondiale.

Q ue recouvre le terme métropolisation qui est entré en usage dans les années 80 ? Jacques Lévy définit le processus de métropolisation comme la tendance de la grande ville à devenir plus grande (Lévy 2000). Cette aporie rend compte tout d'abord de la prise de conscience de la faible pertinence des théories du rendement décroissant qui mettaient l'accent sur les externalités négatives engendrées par la grande ville. Elle est associée très directement à l'objet de recherche ville globale qui a dominé la réflexion sur la place des grandes villes dans l'économie mondiale (Sassen 1991), et plus précisé ment dans les recompositions productives et territoriales liées au changement du régime d'accumulation durant ces années-là. Ces analyses sur les processus de métropolisation, dans le droit fil des travaux sur l'accumulation flexible de l'Ecole de la régulation (Lipietz et Boyer) ont soulignél'importance des facteurs d'attractivité et de localisation liés aux fonctions stratégiques et de commandement des grandes villes.

Parmi les conditions qui contribuent à l'attractivité des grandes villes, I'action d'un gouvernement capable de promouvoir une image cohérente et de mettre en place de nouvelles formes de gouvernance est décisive.

\footnotetext{
${ }^{1}$ "Aujourd'hui, on ne peut ignorer cette évidence. Ce sont les villes qui déterminent le plus ou moins grand niveau de compétitivité des pays, dans un climat de compétition entre les villes et les pays où il y aura des gagnants et des perdants" (déclaration de B. Pinto, maire de Rio, 1999).
} 
L'apparition, dans les années 80 , du terme de gouvernance, - terme qui vient de la science politique-, envahit peu à peu le champ de l'urbanisme et des études sur la ville ainsi que de l'expertise territoriale ou encore l'économie régionale. Le succès que l'usage de ce terme a connu s'est accompagné d'une certaine confusion. Pour la sociologie économique, l'usage de cette notion a pour vocation de rendre compte des formes différentes d'enracinement et de régulation de l'économie, dans les pays capitalistes, c'est-à-dire de prendreen compte les conflits de pouvoir, le jeu des groupes sociaux et les mécanismes de contrôle. Après l'énonciation très en vogue au début des années 80 de l'idée de crise urbaine, il s'est agi de mettre en évidence les formes horizontales d'interaction entre les acteurs et leurs interdépendances dans toute leur complexité. La gouvernance n'a pas remplacé le gouvernement qui garde ses capacités d'arbitrage. M ais l'intérêt de cette approche est de mesurer les changements dans les modes d'articulation entre le public et le privé. Elle permet d'aborder les logiques de recompositions de l'Etat et les nouvelles relations entre pouvoir et territoires, dansun processus de mondialisation et d'intégration à l'échelle du continent et de mettre l'accent sur la multiplication des acteurs, étatiques ou non.

Aussi, un des défis majeurs que les dispositifs de gouvernement ont eu et ont à relever est de parvenir à concilier les objectifs de performance urbaine avec ceux de construction d'une légitimité locale.

Pour autant, Saskia Sassen avancel'idée que la villeglobaleconstitue un espace privilégié pour de nouvelles formes d'action politique. En Amérique latine, de Bogota à São Paulo, en passant par Curitiba et Porto Alegre, les villes ont été le lieu d'innovations politiques et deformesd'action collectivequi ont largement contribuéà leur transformation. Toutefois dans le cas de la ville de Buenos Aires, peut-être plus dans toutes autres villes del'Amériquelatine, la quête demiseen place de nouvelles formes de gouvernance a été confrontée à des processus d'hétérogénéisation de l'espace, à un accroissement des inégalités socio-spatiales et à la montée du chômage et de la pauvreté.

$D$ ans cet article, je voudrais mettre l'accent sur la fragmentation institutionnelle au sein de l'espace métropolitain, afin de saisir, en fonction des temps politiques et des territoires, les bifurcations et les "styles de gouvernance" qui opposent et/ou déconnectent la ville-centre des municipalités de la banlieue. Partant de mon expérience de recherche dans le Grand Buenos Aires, initiée depuis le début des années 80 , cette analyse réflexive sorganise autour des trois questions suivantes. Q u'entend-t-on par gouvernement local dans une grande métropole? Q u'en est-il de la participation et de ses avatars? Enfin quelles sont les échelles de l'inscription territoriale de l'action collective? 
A la différence dela science politique, l'approche géographie qui sera la nôtre, ne place pas au centre de l'analyse la question de la marge d'autonomie des autorités locales au sein de l'Etat, mais plutôt celle de la capacité des territoires à devenir des acteurs collectifs de la gouvernance, à peser sur leur devenir et en particulier à intégrer les citoyens à la ville.

\section{Une lecture institutionnelle de la ville: Ordre politique et régimes des idées dans l'espace métropolitain}

Tout d'abord, que signifie parler de gouvernement local, d'initiatives locales, dans une grande métropole de 12 millions d'habitants caractérisée par une coupure radicale entre la ville - centre (3 millions d'habitants) et les périphéries? S'agit-il de penser le gouvernement et la gouvernance au niveau de ce vaste ensemble divisé juridiquement, entrele gouvernement dela ville de BuenosAires et les 24 municipalités de la banlieue sous tutelle de la province du même nom. La fragmentation institutionnelle est sans aucun doute l'aspect le moins ambigu de la fragmentation urbaine. Car la manière dont sont construits les territoires du politique représente une dimension incontestable d'une gestion urbaineà plusieurs vitesses, entrela capitale, une île du premier monde, progressiste, - à tout le moins qui se pense comme telle -, et des banlieues certes composites, mais dans leur immense majorité appauvries et restées fidèles au péronisme.

Ici comme ailleurs, l'idée métropolitaine vient buter contreles frontières à l'intérieur desquelles se sont construits des territoires politiques. $D$ ans ces limites se sont développés des styles de gouvernement local qui résultent des rapports de force entre les différents ordres de gouvernement, en fonction des temps politiques et du régime des idées qui les portent. $D$ ans une large mesure, les formes de gestion locales sont surdéterminées par les configurations spatiales de la métropole et la position de ses différentes composantes dans le système fédéral argentin, mais elles sont aussi fortement réactives aux dynamiques sociales et politiques de ces mêmes territoires. $D$ ans lesystèmeélectoral argentin, les banlieues de Buenos Aires sont doublement marginalisées et donnent aux acteurs politiques des municipalités des capacités réduites de peser sur la dépense publique en faveur de cet espace déshérité (E scolar et al. 2001).

A l'évidence, la vision d'une ville unifiée par la coordination gestionnaire des acteurs que prétend mettre en place la gouvernance soulève bien des questions.

A quelle échelle doit-on penser la gestion urbaine démocratique, associée à l'idée de décentralisation et de participation? Entrel'échelle du quartier et celle dela métropole, quels sont les niveaux pertinents pour l'exercice de la démocratie local e? Comment créer des instances de coopération permettant de prendreen compte la dimen- 
sion métropolitaine, sans laquelle ne peuvent être véritablement entendues les demandes de l'ensemble des habitants?

A Buenos Aires, ce questionnement est ancien. II apparaît avec l'expansion accélérée de l'agglomération, au tournant du siècle. $D$ ans les années trente, la dimension métropolitaine dela villea étéplaidéepar les partisans d'un plan d'extension (ensanche) qui permettrait d'établir une relation organique entre la ville et les nouvelles urbanisationspériphériques. $M$ ais, la construction durant ces mêmesannées del'avenue G eneral Paz, sorte de boulevard de ceinture, est venue matérialiser la coupure entre les deux espaces. D epuis lors, demanière insistante, les maux del'agglomération, en particulier la fracture entre la ville et les banlieues, mais aussi les problèmes de transports, de contrôle des inondations, des équipements hospitaliers ont été imputés à l'absence d'une instance métropolitaine et de dispositifs de coordination.

D ans cettemétropoledivisée, les banlieues appauvries et la ville-centreont connu des trajectoires politiques, à la fois décalées par rapport aux temps politiques et enfermées dans leur territoire. M ais dans un cas comme dans l'autre, le tournant libéral des années 90 marque une forte inflexion dans les modes de gestion de ces territoires. La privatisation des services urbains et denombreux espaces publics, le retrait de l'Etat, l'autonomie municipale acquise de la villecentre en 1996 et la reprise en main par la province de Buenos Aires des communes de la périphérie ont eu pour effet d'accentuer les contrastes au sein de l'espace métropolitain.

Ces contrastes sont d'autant plus forts que la régulation urbaine est désormais déléguée, dans une large mesure, à des opérateurs extérieurs au champ politicoadministratif. Aussi voit-on clairement se mettre en place un principe différencié de la gestion del'espace. D 'un côté, lesstakehol ders, terme boursier forgépar les organismes internationaux pour désigner ceux qui sont partie prenante dans l'organisation de la ville, des actionnaires, c'est-à-dire, les grands opérateurs de services urbains privatisés (Aguas Argentinas, Edenor, Edesur, etc.) et les grands ensembliers (deve loppers) qui pèsent d'un poids considérable dans les réorganisations territoriales. Les négociations actuelles entrent les privati sées des services urbains et le gouvernement pour la révision des tarifs soulignent, s'il en était besoin, que les logiques et les temps del'entreprise ne sont pas ceux du service public ${ }^{2}$. D el'autre, les autorités locales, les $O N$ G, les associations d'habitants et l'É gl ise qui, toutes à leur manière, développent des politiques sociales territorialisées dans les "zones défavorisées" du Sud de la capitale et dans les périphéries. $C$ ar, on ne peut pas réduire la métropole à la ville-centre

\footnotetext{
"Autorizán a las privatizadas a bajar la calidad de los servicios", Clarin, 25 août 2002.
} 
qui s'étend sur un peu moins de $200 \mathrm{~km}^{2}$, concentre $25 \%$ du PIB du pays, et dont les habitants avaient, jusqu'en décembre 2 001, un revenu moyen de plus 20000 dollars/an (presque troisfois supérieur à la moyennenationale), tandis que plus d'un tiers des habitants de la périphérie vivait au-dessous du seuil de pauvreté. C'est dans ces zones que les autorités locales en partenariat avec les acteurs émergents cherchent à mettre en place des formes de synergies - d'empowerment pour reprendre le terme en vogue aux États-U nis - pour faire face aux problèmes de pauvreté, de chômage et d'exclusion (Schapira 2000). Il en est résulté un durcissement de la frontière entre la villecentreet les périphéries, et destrajectoires opposées dans les formes de gouverance.

\section{Buenos Aires: la nouvelle gouvernance des années 90}

En effet, dans les années 80, la ville de Buenos Aires encore sous tutelle de l'Etat, était restée largement à l'écart de la dynamique participative qui avait touché les municipalités de la banlieue, notamment les municipalités les plus pauvres de la deuxième couronne. A contrario, dans les années 90 , les grandes manœuvres spéculatives qui livrent la ville aux developers provoquent en réaction un mouvement citoyen qui débouche sur l'autonomie de la ville (1996). II n'est pas question ici de développer plus à fond les changements qui ont eu lieu à Buenos Aires, dans une ville dopée par les investissements étrangers et en pleine effervescence politique (Schapira, 2000). Je voudrais seulement souligner la force des modèles venus d'Europe dans la construction de la gouvernance urbaine. Trois villes, Barcelone, Bologne, Bilbao, ont inspiré, à des degrés divers, le changement politique et urbain.

Tout d'abord, c'est le modèle Barcelone qui imprime fortement sa marque à la nouvelle gouvernance urbaine des années 90 . La requalification des anciens docks de Puerto $M$ adero est sansaucun doutela réalisation la plus emblématique des grands chantiers urbanistiques et du city-marketing. Les changements qui ont lieu l'ont été dans une étroite relation avec la capitale catalane, en la personne de Jordi Borja qui a exporté, ici comme dans de très nombreuses villes d'Amérique latine, de Belem à Val paraïso, son plan stratégique. M ais le virage idéologique de l'équipe à la tête du projet change la nature de l'opération. Car à la différence de Barcel one où les effets du projet urbain d' "actualisation de la vieille ville et de sa mise en tourisme" dépassent largement lecadre del'ensanche, à Buenos Aires les actions et investissements se sont concentrés entre Puerto $M$ adero et la C ostanera $N$ orte, c'est-à-dire dansla partie la plus riche de la ville, sans souci de rééquilibrage. $D$ ans ce cas, on retient du modèle le projet architectural et non les équipements et l'amélioration des réseaux.

$N$ ous avons vu que ces opérations qui ont donnélibre cours aux affaires ont fait surgir en réaction des mouvements d'habitants pour la défense de l'espace public et 
contre les privatisations. Ces mouvements de classes moyennes, d'intellectuels progressistes qui ont alors porté le changement politique regardent vers la ville de Bologne (Schapira 2001). En effet, dès les années 70, dans les milieux critiques del'autoritarisme planificateur, le modèle d'intervention de la ville euro-communiste de Bologne avait été très présent dans la réflexion sur la réhabilitation des centres et la démocratie locale. C'est pourquoi Bologne a été le référent pour penser le futur découpage en communes inscrit dans la nouvelle Constitution de la ville (1996). La création de communes disposant de compétences territoriales et d'un gouvernement élu au suffrage universel direct est adoptée pour permettre aux organisations de la société ci vile (terme employé couramment pour parler des dynamiques sociales de la capitale, à la différence des municipal ités de banlieue), de tranfformer le vieux système bureaucratique de la mairie de Buenos Aires, en un modèle à la fois plus efficace et plus démocratique ${ }^{3}$. Toutefois, la mise en œuvre de la décentral isation intra-urbaine qui supposait un reformatage des territoires politiques a profondément diviséla nouvelle majorité. Elle a opposé les défenseurs de l'idée de la ville comme un tout, véritable échelle d'un débat citoyen, dénonçant l'ambiguïté d'une réforme qui occultele poids pris par les grands opérateurs et laisse les voi sinssans voix, aux partisans d'une échelle de représentation plus proche des habitants. Ces tensions et enjeux politiques ont bloqué la réforme communale qui s'est limitée jusqu'à maintenant à la création de 16 "C entres de Gestion et de Participation" (CGP). A travers ces postures opposées s'exprime la tension toujours forte entre voisins et citoyens, et entre deux échelles d'action et de représentation, les quartiers et la ville.

Enfin, lorsque le gouvernement de la ville autonome décide de lancer une action lourde en direction des vieux quartiers industriels et populaires du sud de la ville, en déshérence, Bilbao est présentée comme une expérience à suivre pour requalifier cet espace. Unedes actions envisagées par lenouvel organisme crééà cet effet, la Corporación Sur s'inspire de la recette Bilbao. Elle reprend l'idée de la construction d'un Guggenheim par un architecte du star system qui donnerait le signal de la renaissance du Sud. M ais ce projet comme les autres ont été emportés dans la débâcle argentine de l'année 2000.

Avec la crise, le paysage dévastéqu'offrela capital ejusque dans ses partiescentraleslaisse voir unevilleabandonnéedurant les années 90 , par l'action publique. La nouvelleformede gouvernance de la période de la convertibilité reposait avant tout sur une articulation entre lesintérêts privéset ceux du gouvernement M enem. Pendant toutela dernière décennie, les investissements publics ont déserté les quartiers, notamment le sud de la ville.

\footnotetext{
${ }^{3}$ Le mot voisin, est pris ici dans le sens restrictif que ce terme a dans la tradition hispanique, c'est - à-dire de habitants qualifiés propriétaires et contribuables représentant le parti des intérêts locaux, les forces vives.
} 
Les modèles qui ont traversé l'Atlantique ont-ils été dénaturé comme le pense Jordi Borja (2001) en l'absence d'une conduction politique portant au sein de la société le débat sur la réforme urbaine, à la différence de ce qui se passe aujourd'hui dans les villes brésiliennes?

Force est de constater que l'autonomie de la ville résultant d'une dynamique politique de défense de la puissance publique face à la "privatisation de la ville" ${ }^{\prime \prime}$ n'a pas véritablement entraînéunerupture avec la gestion précédente, celledu ménémiste Carlos Grosso (1989-1992), qui avait livré la ville aux affaires. D ans la deuxième étapedu projet dePuerto $M$ adero, les régulations étatiques sont absentes et l'alliance entre les developers, le gouvernement de la ville et la corporation des architectes organiques perdure. Aussi, I'autonomie acquise est vite apparue comme une arme à double tranchant, enfermant les actions et la pensée sur la ville dans la partie centrale, la plus consolidée. Les nouvelles autorités sont restées, elles aussi, grisées par le discours sur la ville globale, sans projet, sinon celui d'attirer les investisseurs, au coup par coup. N ousnenous sous-estimons pas la volontéde réformes, ni les difficultés deleur application. $D$ eschantiers ont étéouverts, celui dela décentral isation, des politiques culturelles, mais dans l'ensemble, les politiques n'ont pas été à la mesure desambitions affichées et des atouts immenses qu'offrait la ville de Buenos Aires ${ }^{6}$. Le "gouvernement de la ciudad autonoma", - au-delà de quel ques discours convenus a ignoréla réal ité métropolitaine, ou plutôt elle a été avant tout pensée en termes de coût de centralité (utilisation par les banlieusards des écoles, des hôpitaux) et non commeuneopportunité pour développer les équipements et de nouvell es central ités. Aussi durant toute cette période, la coupure s'est accentuée entre la ville et les périphéries de plus en plus renvoyées à elles-mêmes, au fur et à mesure que grandissaient le chômage et la pauvreté.

\footnotetext{
${ }^{4}$ C'est un terme toujours utilisé en Argentine, très largement auto-référentiel.

${ }^{5}$ "Lo que he dicho, es claramente algo que tiene que ver solamente con un número limitado de ciudades. Hay muchos otros discursos sobre ciudades que no tiene que ver nada con lo que he hablado. Pero creo quela Ciudad de Buenos Aires- yme ocurre que esto es parte de lo queimpulsa el proyecto de este congreso - tiene quever con estas posibilidades estrategicas que afectan solamente a una minoridad de ciudades. Creo que Buenos Aires es parte de esa minoridad”. Conférence prononcée par Saskiasassen lors du Colloque international, Hacia una estrategia para Buenos Aires, organisé par le Gobiemo de la Ciudad de Buenos Aires, 28 et 29 juillet 1997.

${ }^{6}$ Les CGP ne sont restés que des antennes de la municipalité et non de véritables instances de participation et de discussion. La Corporacion Sur n’a pas attiré d'investissements dans le Sud.
} 
Tableau 1

Evolution de la pauvreté dans l'agglomération de Buenos Aires (1991-2001)

Année

1991

1992

1995

1998

1999

2000

2001 ville de Buenos Aires pauvres

$8,10 \%$

$5,60 \%$

$8,00 \%$

$5,90 \%$

$8,30 \%$

$9,50 \%$

$9,80 \%$

source: INDEC, EPH, octobre 2001 municipalités du Conurbano pauvres indigents

$26,40 \%$

$3,80 \%$

$22,40 \%$

$4,00 \%$

$30,50 \%$

$7,90 \%$

$32,40 \%$

$7,90 \%$

$32,50 \%$

$8,40 \%$

$35,00 \%$

$9,50 \%$

$43,20 \%$

Carte $n^{\circ} 1$

Les disparités socio-spatiales dans le Grand Buenos Aires

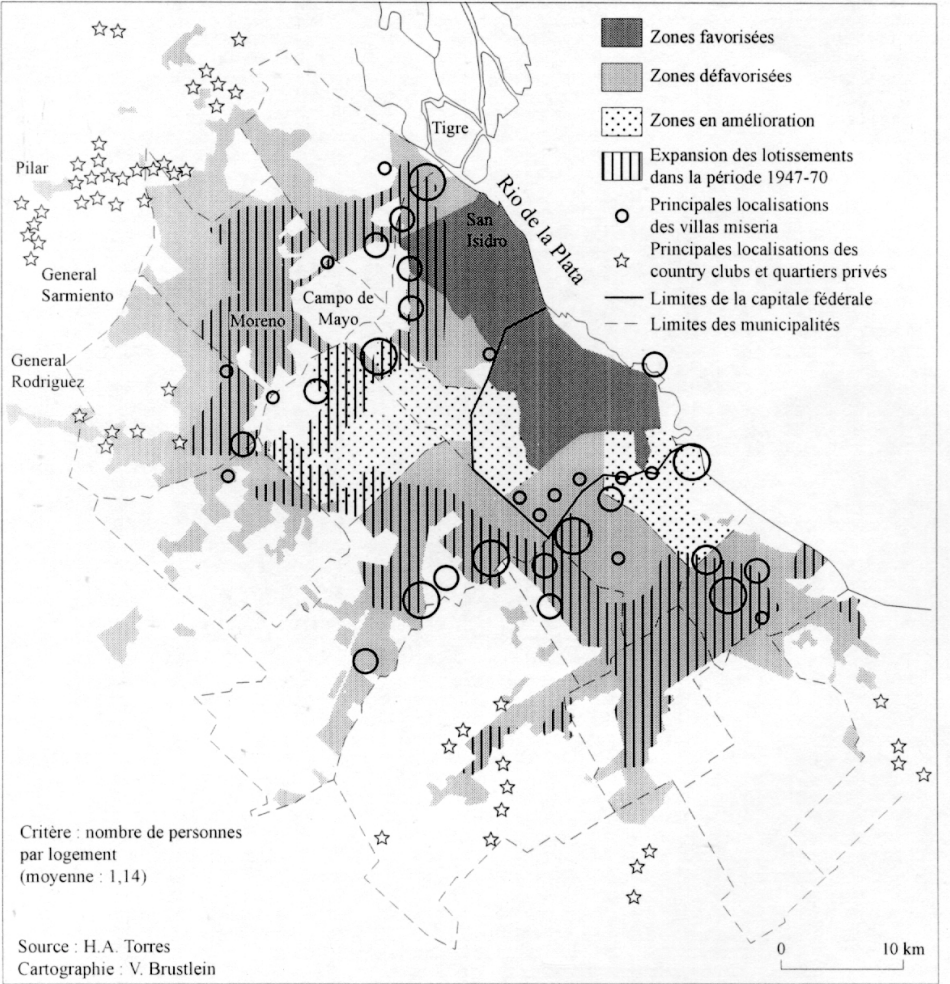




\section{Les banlieues: de l'utopie au pragmatisme ou les avatars de la participation}

La décennie de la convertibilité (1991-2001) s'est terminée comme elle avait commencé par des émeutes et des pillages dans les banlieues appauvries des grandes villes argentines, notamment dans le Conurbano bonaerense. La crise terminale a frappé de manière visible et violente ces espaces de la périphérie, laissés à l'écart des investissements étrangers directs et les grandes opérations urbanistiques concentrés dans la ville. Elle a rappelé la puissance destructrice des politiques néo-libérales mises en place dans les années 90 . $D$ ans les périphéries de Buenos Aires, dans pratiquement tous les domaines, les institutions laissent des pans entiers de la population et de vastes zones dans le désordre et l'instabilité, que ce soit au niveau de l'éducation, de la santé, des transports, des services urbains et du logement. Rien ne fonctionne plus, ou presque, dans certains quartiers de la périphérie. Les événements de décembre 2001 ont fait éclater au grand jour la tension entre d'un côté, les processus de métropolisation sélective, visant à l'excellence et à la participation à des réseaux de commandement mondialisés, et de l'autre, l'approfondissement des fractures internes au sein de la grande métropole argentine (Schapira 2003). Enfin, ces années ont été marquées par un recul des formes de participation au niveau local qui avaient caractérisé le retour à la démocratie.

En effet, durant ces années, les banlieues de Buenos Aires avaient été le lieu d'expériences et d'innovations locales impulsées par la dynamique politique de la transition démocratique. Plusieurs questions avaient alors induit le débat sur le local et la participation. Comment rétablir un système démocratique qui assure le plein usage des libertés publiques et une plus large participation des secteurs à bas revenus? Comment éviter l'exclusion d'une part croissante de la population dans un pays qui connaît dans les années 80 une forte paupérisation? Comment gérer la balkanisation des territoires de la ville et introduire de l'efficacité et de la synergie dans des espaces sous-équipés et sous-administrés?

Rappelonsque, en dépit des références à la décentralisation, au local et à la participation qui ont envahi le discours des acteurs urbains, des militants comme des politiques dans des années 80 , il n'y pas eu de réforme décentralisatrice dans la province de Buenos Aires, à la différence d'autres provinces argentines (Cordoba, Santa Fé). D e fait, les municipalités du Conurbano ont une faible consistance institutionnelle et nejouent pas véritablement le rôle de gouvernement local. Elles restent sous tutelle du gouvernement dela provinceet nedisposent, pour beaucoup d'entreelles, d'aucune autonomie financière. Plus de $90 \%$ de leur budget est absorbépar les dépenses courantes. Elles peuvent difficilement imposer des règles et des normes à l'action publique 
locale et apporter des réponses concrètes aux difficultés quotidiennes. Ce sont avant tout des lieux demobilisation et d'accumulation politique dansun espace où plusd'un tiers des él ecteurs argentins se trouvent rassemblés. $D$ ans ces territoires, lemaillage des partis, des associations de quartier, de l'Eglise structurent des appartenances, des solidarités et des identités sur lesquelles les différents acteurs se sont appuyés pour mettre en œuvre des projets, des formes d'actions collectives et de participation politique.

Toutefois, avec le retour à la démocratie, les municipalités de la périphérie ont été en première ligne, donnant force à une institution longtemps restée subalterne. Après sept années de dictature (1976-1983), c'est à ceniveau de proximité relégitimé par le rétablissement des élections que, se sont adressées les demandes des populationsles plus démunies. A cetteéchellese sont affirmés denouveaux acteurs- Eglise(s), O N G , organisations populaires - , obligeant les pouvoirs locaux à s'impliquer plus directement dansla gestion de leur territoire. C'est dans ce contexte, quelesmunicipalités les plus pauvres, celles de la deuxième couronne, ont voulu faire de l'exercice municipal le "banc d'essai" de projets qui renouaient avec les idées basistes. Ce courant fortement influencé par les idées de la théologie de la Libération, del'Église des pauvres, a cherchéà mettre en place des expériences productives et organisationnelles pour donner autonomie et dignité à la communauté, des expériences fondées sur la solidarité territoriale du quartier, du lotissement ou de la paroisse et sur la participation de la population.

$D$ ans une approche communautaire du territoire, il s'est agi de valoriser les initiatives locales, de prendre en compte les cultures, les solidarités et les savoirs faire populaires. $D$ ans ce cas de figure, le quartier est appréhendé comme base de la mobilisation et de l'action, afin d'utiliser les ressources de la proximité. En reprenant l'idée de D. Béhar et Ph. Estèbe (1995), on peut parler ici d'une conception ethnographiquedu territoire "Chaque territoire devient en quelque sorte une concession expérimentale" devant permettre à terme un changement global. Cette approche part du présupposé que la proximité sociale et géographique constitue un facteur puissant d'incitation à l'action. Pour analyser ces dynamiques participatives, il faut bien sûr les replacer dans le climat de la transition politique des années 80. Les formes de mobilisation des premières années de la démocratie ont étéchargées d'unecertaine dose d'utopiequi voyait dansces expériences un pas vers "la construction d'un nouveau modèledémocratique" (Schapira 1999). Elles se sont accompagnées de toute une réflexion sur les échelles de

${ }^{7}$ Le basisme est né à la fin des années 60 de la convergence singulière d'un courant religieux proche de la théologie de la Libération et d'un mouvement séculier, le péronisme. 
la participation, sur la nécessité de créer de nouvelles instances délibératives, les "conseils de la communauté" regroupant plusieurs quartiers.

Je ne reviendrai pas ici sur toute une série d'expériences, de projets et d'innovations en matière de gestion urbaine qui ont vu le jour durant ces années de la transition: grands mouvements d'occupations de terre (asentamientos), coopératives de logement, de services, petite production artisanale. D ans tous ces projets, il faut souligner l'importance de la circulation des idées au niveau de l'Amérique latine d'une part, et d'autre part entre les ONG et les organismes internationaux. Car durant ces mêmes années, les idées basistes entrent en résonance avec les idées libérales des bailleurs de fonds. En prônant la communautarisation des politiques sociales (grass root planning), la Banque mondiale récupère les formes d'actions qui ont été celles des mouvements proches de l'Égl ise des pauvres, de certains mouvements révolutionnaires, des ON G, pour les mettre au service de sa logique d'intervention . Les populations sont engagées à participer aux programmes mis en œuvre par la Banquemondiale, en fournissant un apport en travail ou en argent. La notion d'implication est à la base de ces projets pour défendre la dignité mais aussi parce que les solidarités communautaires sont appelées à prendre le relais des initiatives et des financements del'État. Ces programmes d'entraide, auto-construction, d'aidealimentaire sont ciblées sur les zones de pauvreté. Ils concernent avant tout les conditions de vie des habitants, - logements, infrastructures -, car de plus en plus les droits sont revendiqués sur la base de l'appartenance à un quartier, à une communauté circonscrite à un territoire. $\mathrm{C}$ ar en Amérique latine, le terme communauté prend des sens bien différents selon qu'il se rattache aux formes coprophagies de l'Etat (la communauté organisée de Peron), au vocabulaire de l'Eglise des pauvres ou encore à la communauté paysanne traditionnelle. Bref, la polysémie du terme est forte mais pour les bailleurs de fonds comme pour les réformateurs sociaux: cadres des municipalités, travailleurs sociaux, O N G toujours plus présentes dans la ville latino-américaine, militants politiques ou associatifs, la communautédevient un concept opérationnel qui doit permettre de définir un territoireauquel est reconnu lestatut decommunauté. $C^{\prime}$ 'est sur cette base que les pauvres urbains font reconnaître leurs droits aux nouvelles formes d'action publique.

\footnotetext{
${ }^{8}$ Le régime militaire d'Ongania a mis l'accent sur le développement de la communauté. En 1971, il existait un réseau national de développement communautaire rattachéau département du développement des communautés de la Direction générale de la promotion communautaire.
} 


\section{Le quartier et l'inscription territoriale de l'action collective}

Si dans les premières années de la démocratie, l'arène municipal eavait été pour les militants et les partis l'espace de la participation, avec l'approfondissement de la crise et les effets des politiques d'ajustement, elle devient avant tout comme l'échelle de la mise en place des politiques assistencielles (Schapira 2000). D ans un contexte d'appauvrissement et de démantèlement de la réglementation étatique, s'est al ors opérée par le biais des bailleurs de fonds, la convergence entre deux traditions, deux démarches, l'une de solidarité latine, de type clientéliste et national-étatique, l'autre d'assonance anglo-saxonne inspirée par les préceptes de la Banque $M$ ondiale enjoignant les gouvernements à dével opper des politiques communautaires à l'égard des pauvres. II va de soi que ces préceptes viennent se greffer sur l'espace social de villes qui ont leur propre histoire et dont les évolutions ne peuvent pas être lues de la même manière.

$D$ ans le cas qui nous intéresse, le tournant idéologique du début des années 90 et la banalisation du discours libéral au sein même du péronisme militant, sur fond de crise et de pauvreté croissante, mettent à mal les dynamiques participatives. Alors que la capitale accède à l'autonomie, les municipalités sont reprises en main par le gouvernement provincial et ses réseaux politiques. Le recul brutal desformes d'organisations dela période antérieure soulignela fragilité de ce quej'ai appelé “la décentralisation de fait", c'est-à-dire de dynamiques sans reconnaissance et appui institutionnels. L'observation sur la durée montre que ces solidarités de quartier ne peuvent fonctionner sans médiations institutionnelles (santé, école, gestion des services urbains), et ce d'autant que ces expériences et initiatives se situent dans une immense banlieue où se sont renforcés, durant la convertibilité des processus de polarisation socio-spatiale symbolisés, d'un côté par l'apparition aussi soudaine que massive des quartiers privés (gated communities) à la lisière dela ville et del'autre, l'augmentation desvillasmiseria (bidonvilles) et occupations deterre(asentamientos), dansune grande proximité (Schapira 2000).

D ans les années 90 , dans les quartiers de la périphérie, le climat a été marqué par un "sentiment de déroute" pour reprendre l'expression de L. Kowarick. Toutefois, le dynamisme de la participation des années 80 y a laissé de fortes empruntes.

Q uelles sont-elles? Un style d'expériences dont on pourrait multiplier les exemples, - coopératives de logements, de servicesb, micro-crédits, qui ont donné une place croissante au quartier. La présence de l'E glise dans les quartiers pauvres de la périphérie a largement contribué à l'inscription territoriale del'action collective et à l'émergence de leaders territoriaux qui tirent leur force et leur capacité de négociation avec le système politique, de leurs bases locales. C es expériences qui réaffirment le droit à la terre, le droit au logement, le droit au travail ont inspiré les politiques 
urbaines nationales ou locales, comme ce fut le cas de la régularisation des terres publiques occupées (le plan Arraïgo) ou encore, des terres vacantes dans la province de Buenos Aires (loi Pierri).

Enfin, les nouveaux répertoires d'action collective qui sont apparus ces dernières années, puisent largement dansces expériences. Les mobilisationsqui sorganisent pour l'accès aux bénéfices des politiques social es de lutte contre la pauvreté sappuient avant tout sur les organisations de quartier. Cette politique "d'assistance participative" occupe désormais une énergie considérable dans la vie des plus pauvres C'est sur la base du quartier que les familles démunies (carenciadas) ont accès aux politiques sociales et qu'elles sont insérées dans des réseaux politiques, religieux et associatifs. La multiplication des affiliationsau niveau local fonctionne commeune assurancesocialepermettant de faire face aux risques permanents du présent (M erklen 2002).

L' importance du quartier est à souligner dans les dynamiques décrites. En Amérique latine, le mot prend un sens bien différent, selon les pays. Si au Venezuela, le mot barrio évoque d'entrée de jeu des zones pauvres et précaires, en Argentine, le terme est associé à l'idée d'intégration à la ville et à la citoyenneté.

C'est pourquoi dans ces banlieues dévastées, le quartier et sa construction permet d'unifier des répertoires d'action collective divers d'une population qui forme un monde extrêmement hétérogène organisé par de nombreux réseaux, une trame qui se structure à l'échelle locale. $D$ enis $M$ erklen parle de réaffiliation au niveau du quartier. Le développement des organisations des coupeurs de route (pi queteros) formées de chômeurs et l'appropriation de la méthodologie du piquete de la part de centaines d'organisations de quartiers dans les périphéries de la métropole résultent decettefaçon defaire dela politique. Cettefaçon defaires'est construitedepuis plus dedix ans, autour des réseaux de l'Eglise et des partis, notamment du parti péroniste qui irrigue de manière dense le territoire du Conurbano. Les principaux dirigeants du mouvement piquetero sont issus pour beaucoup de celui des occupations de terre des années 80 et du combat mené pour le "droit à la ville", soulignant ainsi la filiation et la capitalisation des expériences passées. Les organisations piqueteras se placent dans le jeu entre le local et le national, court-circuitant l'échelon municipal et les réseaux clientélistes des punteros, (version argentine du boss de quartier). Elles jouent un rôle grandissant dans l'attribution des plans sociaux dont elles sont les gestionnaires dans les territoi res qu'elles contrôlent. 


\section{Que peut-on retenir de cet exercice réflexif sur les styles de gouvernement local dans l'agglomération de Buenos Aires?}

- Tout d'abord, il apparaît indispensable pour la recherche de penser ces expériences dans la durée, de reconstruire la mémoire des mouvements urbains pour en comprendre leur dynamique politique et sociale et leur inscription territoriale. A travers cet exemple, il est difficile d'identifier des forces qui portent un projet urbain cohérent, durable, soulignant la forte fragmentation des différents acteurs qui agissent dans des sphères et des échelles différentes. Entre l'échelle étatique et les micro projets, existe-t-il une échelle intermédiaire de bonne gouvernance?

- En second lieu, il convient de souligner l'importance des territoires du politique dans l'analyse des formes de mobilisation et de participation, soumises "aux hauts et aux bas" de la relation entre l'associatif et l'institutionnel. Ces formes d'empowerment se déroulent dans un contexte de privatisation et de retrait de l'Etat qui voit s'instaurer de nouveaux découpages - ceux des privatisées, en fonction des clientèles et de I'encapsulage des pauvres pour reprendre lestermes des. Jaglin, et des acteurs multiples qui sont désormais partie prenante de l'urban governance. Parmi eux, les organismes internationaux, les grands opérateurs de services urbains privatisés et les grands ensembliers (developers) pèsent d'un poids considérable dans les réorganisations territorial es. En Argentine, les tensions actuelles entre les privatisées des services urbains et le gouvernement pour la révision des tarifs soulignent, s'il en était besoin, que les logiques et les temps del'Entreprise ne sont pas ceux de l'action publique ${ }^{9}$.

- En troisième point, quel regard porter sur toutes ces expériences participatives? II apparaît nécessaire de replacer ces best pratices, dans une échelle plus vaste, celle de la métropole, car pour reprendre l'idée de M arc Lévy “nous ne sommes pas en manque d'actions exemplaires. Le problème c'est de leur donner de l'ampleur" (Lévy 2000). Comment se situer par rapport aux interprétations qui parent ces mouvements d'habitants de toutes les vertus démocratiques et de solidarité, sans en souligner à la fois la diversité, sans les penser dans la durée, sans en préciser les objectifs. Les associations de classes moyennes, de riverains propriétaires et celles de quartiers populaires ne défendent pas les mêmes objectifs. Les premières, dans la ville de Buenos Aires, ont joué un rôle important dans le défense de certains thèmes: la démocratie locale, les espaces verts, la culture, la rénovation des centres-villes à l'abandon, les droits de l'H omme. Les secondes, même si elles n'ont pas ignoré ces thèmes ont été dominées par les problèmes de survie, de travail et de logement.

\footnotetext{
9 "Autorizán a las privatizadas a bajar la calidad de los servicios", Clarin, 25 août 2002
} 
- Enfin, dernier point, peut-on penser Buenos Aires sans les banlieues?

La crise a rappelé à la fois la coupure sociale radicale entre la capitale et les banlieues, mais aussi, l'importance de la dimension métropolitaine et des relations fonctionnelles entre la villecentre et le Conurbano. C'est un vaste et même marché du travail. Les cartoneros qui débarquent chaque soir dans la capitale, par le train $M$ itreviennent desvillasmi seria installéeslelong du Rio Reconquista ${ }^{10}$. Plus généralement, c'est environ un million de banlieusards qui vient, chaquejour, travailler dans la ville-centre. La crise financière porteña a immédiatement rejailli sur l'emploi dans la périphérie. M ais, ici, à la différence de la capitale, la pauvretéet le chômage sabattent, de manière différenciée, sur des espace appauvris où les taux de pauvreté même au plus fort de la reprise ne sont jamais tombés au-dessous de $20 \%$, où des quartiers entiers sont sans infrastructures, sans transport public, sans réseau d'eau potable et d'assainissement, un espace confronté au sous-emploi massif depuis 1996. Car durant la convertibilité, au regard des changements économiques et politiques que connaissait la ville, les banlieues sont apparues plus lointaines, prises dans les mailles d'une gestion assitencielle et clientéliste (Plan Vida, Plan Trabajar, Plan Barrios bonaerense, bolsa de comida). En février 2000, Les habitants de la capitale ont vu défilé avec stupéfaction les colonnes de piqueteros venus des communes pauvres du Grand Buenos Aires (La M atanza, Florencio Varela,...), encadrés dans de puissantes organisations, pour demander du travail et des plans d'aide sociale.

D urant toute cette période, la pauvreté et les inégalités ont évolué de manière distincte entre ces deux espaces. La reprise économique a été plus lente dans le Conurbano où la pauvreté atteint son point le plus bas en 1994, pour augmenter de nouveau à partir de 1996 et grimper de manière très significative, à partir de 1998. $M$ ais même lorsque la pauvreté est à son point le plus bas, elle est très nettement supérieure à celle de la capitale, de même que l'on note de forts écarts entre les municipalités de la première et la deuxième couronne. En revanche, durant les années de croissance, les inégalités augmentent et le moteur des inégal ités territoriales se situe dans le corridor nord de la ville. Elles apparaissent davantage comme le fait d'une concentration de la richesse dans certains espaces que de l'appauvrissement des communes pauvres. Autrement dit, l'inégalité est une des facettes du développe-

\footnotetext{
${ }^{10}$ Les techniciens de l'entreprise privée d'électricité Edenor avaient nommé cette zone "la bande de Gaza”. Car lorsqu'ilsy étaient entraient, en 1997, pour régulariser les branchements clandestins, ils avaient étéreçus par les habitants des villas à coups de pierre. Interview du directeur Edenor, Marc Riutort, août 1997.
} 
ment, aussi la récession affecte-t-elle plus violemment les espaces les plus riches, c'est-à-dire la capitale qui présente les plus hauts niveaux de richesse et d'inégalités.

En ce qui concerne la géographie de la pauvreté, le constat est double. O n observe sur la durée une hyperconcentration de la pauvreté dans certaines zones - les poches de pauvreté -, mais en période de récession, ce n'est pas là qu'elle augmentele plus vite. La pauvreté se diffuse dans les quartiers de petites classes moyennes, une pauvretéqui se cache, celles des gasoleros et de ceux qui fréquentent les clubs detroc. Ellene s'inscrit plus seulement dans des périmètres bien circonscrits, celui de la villa, de I'asentamiento ou encore du lotissement à forte proportion de N BI. Or les politiques assistencielles territoriali sées mises en place dans la dernière décennie- le Plan Vida et le système de contrôle par les manzaneras - qui ciblait les "territoires de la pauvreté" ", font l'impasse sur la pauvreté plus diffuse. L'élargissement massif de la pauvreté obligeà mettre en place de nouveaux dispositifs. C'est en partie ainsi qu'il faut comprendre le vaste plan d'aide socialelancé dansl'urgenceen avril 2002, - le plan Jefesy J efas de H ogar - qui cible des individus et non plus des territoires spécifiques.

La brutalité des effets de la crise de l'année 2001 a souligné l'extrême vulnérabilité du modèle des années de la convertibilité, plus spéculatif que productif, soumis au bon vouloir des capitaux et des entreprises privés qui quittent le pays ou abandonnent certains espaces, lorsque le système s'effondre et que diminuent les taux de profit. Elle vient également tempérer le discours sur les vertus de la grande ville, sur sa capacité d'adaptation face à l'incertitude et l'imprévisi bilité d'une économie toujours plus volatile, ainsi que celleà saffranchir du territoire national pour se projeter dans l'espace mondialisé de "I'archipel mégalopolitain mondial”.

\section{Références Bibliographiques}

BÉHAR, D. et ESTÈBE, P. Ville et pauvreté, note bibliographique. Acadie/CDU, 1995.

LÉVY, J. "M etropolisation”, In: Repenser le territoire, un dictionnairecritique. Ed. de I'Aube/D atar, 2000.

LÉVY, M. "Comment renouveler les politiques de coopération au développement"? In: Esprit, juin, 2000. pp. 79-100.

M ERK LEN , D. "Entre ciel et terre: les sciences sociales et la mobilisation populaire en Argentine". In: Les Cahiers des Amériqueslatines, n 41, 2003.

SASSEN, S. The global city. N ew York, London, Tokyo. Princeton, Princeton Universty, 1991. 
SCH APIRA, M .F. P. "Buenos Aires: M étropolisation et nouvel ordre politique”. In: H érodote. $n^{\circ} 101,2^{\text {er }}$ trimestre 2001, pp. 121-151.

. "Buenos Aires: M etropolización y desigualdades". In: EU RE, Santiago del Chile, XXVIII, no 58, 2002, pp.31-50, www.scielo.cl

"D u PAN au plan de justice sociale. Les politiques de lutte contre la pauvreté dans les banlieues de Buenos Aires". In: Les Annales de la Recherche urbaine, $n^{\circ} 86$, juin 2000, p. 133-143.

"From utopia to pragmatism: the heritage of basismo in local government in the greater BuenosAires". In: Bulletin of L atin American Research, Vol. 15, avril 1999.

"Segregação, fragmentação, secessão: a nova geografia social de Buenos Aires". In: N ovos Estudos/CEBRAP, n 56 março 2000, p. 169-183.

"Villes et territoires en Amérique latine: changement de perspective?" in SCHAPIRA, M. F. P. et D 'ARC, H. R. Les territoires de l'Etat-nation en Amérique latine. A Claude Bataillon, Travaux et M émoires de I'IH EAL, 2001.

SVAM PA, M . et PEYRERA, S. Entrela ruta y el barrio. La experiencia de las organizaciones piqueteras. Buenos Aires, Ed. Biblos, 2003, 230 pages.

WELCH , G. M ., BO RJA, J. "Buenos Aires en perspectiva: Berlin y Barcelona”. In: Punto deVista, no 71, 2001, décembre. 